CORRIGENDA

\title{
Molecular cloning, characterization and isolation of novel spliced variants of the human ortholog of a rat estrogen-regulated membrane-associated protein, UO-44
}

Caine Tuck Choy Leong, Chuan Young Ng, Chee Pang Ng, Zeng Shuan Ma, Thanh Hung Nguyen, Sun Kuie Tay and Hung Huynh

Oncogene (2004) 23, 8857. doi:10.1038/sj.onc.1208155

Correction to: Oncogene (2004) 23, 5707-5718.

doi: $10.1038 /$ sj.onc. 1207754

Published online 7 June 2004
The correct list of authors is as below:

Caine Tuck Choy Leong, Chuan Young Ng, Choon Kiat Ong, Chee Pang Ng, Zeng Shuan Ma, Thanh Hung Nguyen, Sun Kuie Tay and Hung Huynh

Since publication of the above manuscript, the authors have identified an error in the list of authors.

\section{Upregulation of $\operatorname{IKK} \alpha / \operatorname{IKK} \beta$ by integrin-linked kinase is required for HER2/neu-induced NF- $\kappa$ B antiapoptotic pathway}

Keishi Makino, Chi-Ping Day, Shao-Chun Wang, Yan M Li and Mien-Chie Hung

Oncogene (2004) 23, 8857. doi:10.1038/sj.onc.1207868

Correction to: Oncogene (2004) 23, 3883-3887. doi: $10.1038 /$ sj.onc. 1207485

Published online 15 March 2004

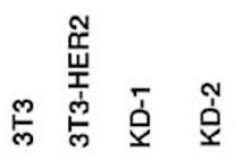

b

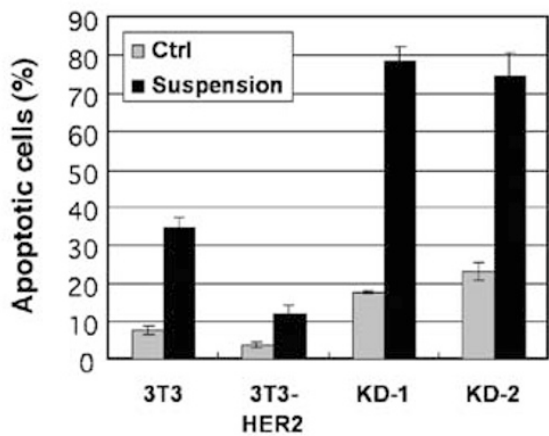

Since publication of the above manuscript, the authors have identified an error in Figure 2. The revised version of the figure is given below.

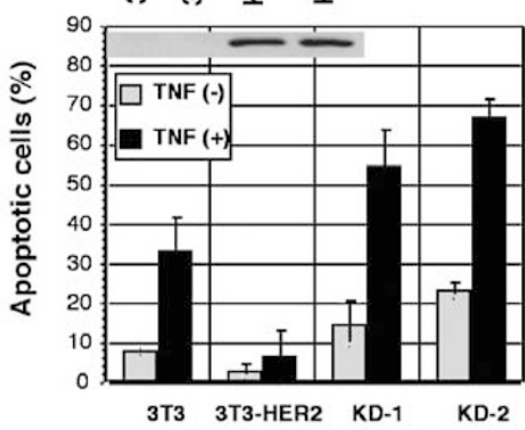

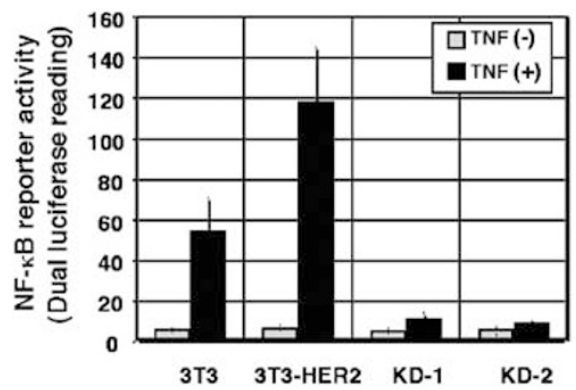

